\title{
How much platelet-rich plasma can be soak-loaded onto beta-tricalcium phosphate? A comparison with or without a unidirectional porous structure
}

\author{
Norihito Arai ${ }^{1}$, Tomokazu Yoshioka ${ }^{1,2}$, Hisashi Sugaya1 ${ }^{1}$, Akihiro Kanamori' ${ }^{1}$, and \\ Masashi Yamazaki ${ }^{1}$ \\ ${ }^{1}$ Department of Orthopedic Surgery, Faculty of Medicine, University of Tsukuba, Japan \\ ${ }^{2}$ Division of Regenerative Medicine for Musculoskeletal System, Faculty of Medicine, University of Tsukuba, Japan
}

\begin{abstract}
Objective: This study aimed to examine differences in platelet-rich plasma (PRP) soak-loaded volumes of $\beta$-tricalcium phosphate $(\beta-T C P)$ with or without a unidirectional porous structure.

Materials and Methods: Leukocyte-rich PRP was extracted from 15 healthy volunteers by centrifugation. Two types of artificial bones were soaked for either ten seconds or ten minutes. The volume ratios of PRP soak-loaded onto the artificial bone and soaked area ratios were evaluated. Statistical analyses were performed using the Tukey-Kramer HSD test and the Games-Howell method. A $P$-value of $<0.05$ was considered statistically significant.

Results: Regardless of the soaking time, the PRP soak-loaded volume ratio and soaked area ratio were significantly higher in the unidirectional porous $\beta$-TCP (UDPTCP) group than in the spherical porous $\beta$-TCP (SPTCP) group.

Conclusion: PRP can be soak-loaded faster and in larger amounts onto UDPTCP compared to SPTCP. Understanding the basic biology of $\beta$-TCP soak-loaded with PRP can help develop more novel and effective $\beta$-TCP treatments for orthopedic surgery.
\end{abstract}

Key words: $\beta$-tricalcium phosphate, platelet-rich plasma, loaded volume, unidirectional porous, artificial bone

\section{Introduction}

Currently, the most common treatment for bone defects is autologous bone grafting. However, this method has several limitations, including the amount of bone that can be harvested, the occurrence of pain at the harvest site, and other issues ${ }^{1,2)}$. Artificial bone, which has excellent osteoconductivity and biocompatibility, is often used as a bone substitute for the reconstruction of large bones with defects and for spinal fusion ${ }^{3}$. However, because non-bone tissues are present in the surrounding area, various attempts have

Received: July 3, 2020

Accepted: September 25, 2020

Correspondence: Tomokazu Yoshioka, Department of Orthopedic Surgery and Division of Regenerative Medicine for Musculoskeletal System, Faculty of Medicine, University of Tsukuba, 1-1-1 Tennodai, Tsukuba 305-8575, Japan

E-mail: tymd99@tsukuba-seikei.jp

This is an open-access article distributed under the terms of the Creative Commons Attribution Non-Commercial No Derivatives (by-nc-nd) License <http://creativecommons.org/ licenses/by-nc-nd/4.0/> been made to promote osteoinduction in artificial bones to promote osteogenesis ${ }^{4-10}$.

The diamond concept, which is used in bone regeneration, proposes four pillars for regenerative medicine using tissue engineering: mesenchymal stem cells, growth factors, scaffolds, and the mechanical environment ${ }^{11)}$.Various tissue engineering methods using soak-loaded cells or growth factors on artificial bone scaffolds have been reported, yet no standard method for scaffold loading has been established. Intriguingly, cells induce osteogenesis when grafted porous artificial bone is soak-loaded with marrow-derived mesenchymal cells ${ }^{5}$. The clinical application of cultured autologous mesenchymal stem cells in bone regeneration has been discussed; however, the allogenic method is preferred because of its convenience, time availability, and cost-effectiveness $^{12}$. Growth factors, especially bone morphogenetic proteins, are the most vital. Unidirectional porous hydroxyapatite (UDPHAp) can load a larger amount of rhBMP-2 and has a higher osteogenic capacity than interconnected calcium porous HAp ceramic (IP-CHA) ${ }^{6}$. However, the use of a high dose of BMP-2 in spine surgery results in ectopic bone formation ${ }^{13)}$. This necessitates the use of autologous 
platelet-rich plasma with growth factors ${ }^{14)}$.

PRP is an autologous product obtained by centrifuging peripheral blood and subsequently separating and extracting the plasma layer, which contains a large amount of platelets. PRP is rich in growth factors involved in osteogenesis, including transforming growth factor- $\beta$ (TGF- $\beta)^{14)}$. In in vitro studies, PRP is involved in bone regeneration where it improves osteoblast chemotaxis, promotes cell growth, and stimulates the autocrine secretion of angiogenic factors ${ }^{15)}$. Additional administration of PRP after autologous bone grafts to mandibular defects promotes osteogenesis more than the autologous bone grafting alone, as evidenced through imaging and histological assessments ${ }^{16}$.

UDPHAp can quickly absorb blood via the capillary phenomenon $^{17}$, and in grafts of tibial defect models, the infiltration of osteoblasts, osteocytes, and capillaries into these pores contributes to the formation of new bone ${ }^{18)}$. In recent years, unidirectional porous $\beta$-tricalcium phosphate (UDPTCP), which also has a unidirectional porous structure, has been developed. UDPTCP can induce osteogenesis as fast as UDPHAp in the same animal model with an improved balance of material absorption ${ }^{19)}$. In grafts used at fibular resection sites in spinal surgeries, UDPTCP results in fewer residual bone defects than spherical porous $\beta$-TCP $(\mathrm{SPTCP})^{20)}$, and the absorption of the material and replacement with a new bone contribute to a strong recovery at the graft site ${ }^{21,22)}$.

$\beta$-TCP plays a role in the scaffold and mechanical envi- ronment, while PRP plays a growth factor. We hypothesized that $\beta$-TCP soak-loaded with PRP would promote osteogenesis in bone defects, where there is little host bone, and in spinal surgery. We assumed that $\beta$-TCP induces osteoclast activity during the bone resorption process ${ }^{23,24)}$. Further, we believed that the growth factors contained in PRP would stimulate the proliferation, migration, and chemotaxis of osteoblasts ${ }^{15)}$, which would promote osteogenesis.

UDPTCP can be penetrated with PRP through the capillary phenomenon, whereas SPTCP can be filled through its spherical pores (Figure 1). Therefore, we investigated whether differences in the structure of $\beta$-TCP affect PRP soak-loaded volumes. To the best of our knowledge, this study is the first to quantify PRP soak-loaded onto UDPTCP.

\section{Materials and Methods}

\section{Ethical approval}

All subjects enrolled in this study provided written informed consent. The study protocol was approved by the Institutional Committee on Human Research at the University of Tsukuba (approval no. 1312).

\section{Subjects}

Fourteen healthy volunteers (mean age, 33.4 years; range, 26-44 years) participated in the study. Inclusion criteria were as follows: Japanese men or women, over 20 years of age. Exclusion criteria were serious medical history, ill-

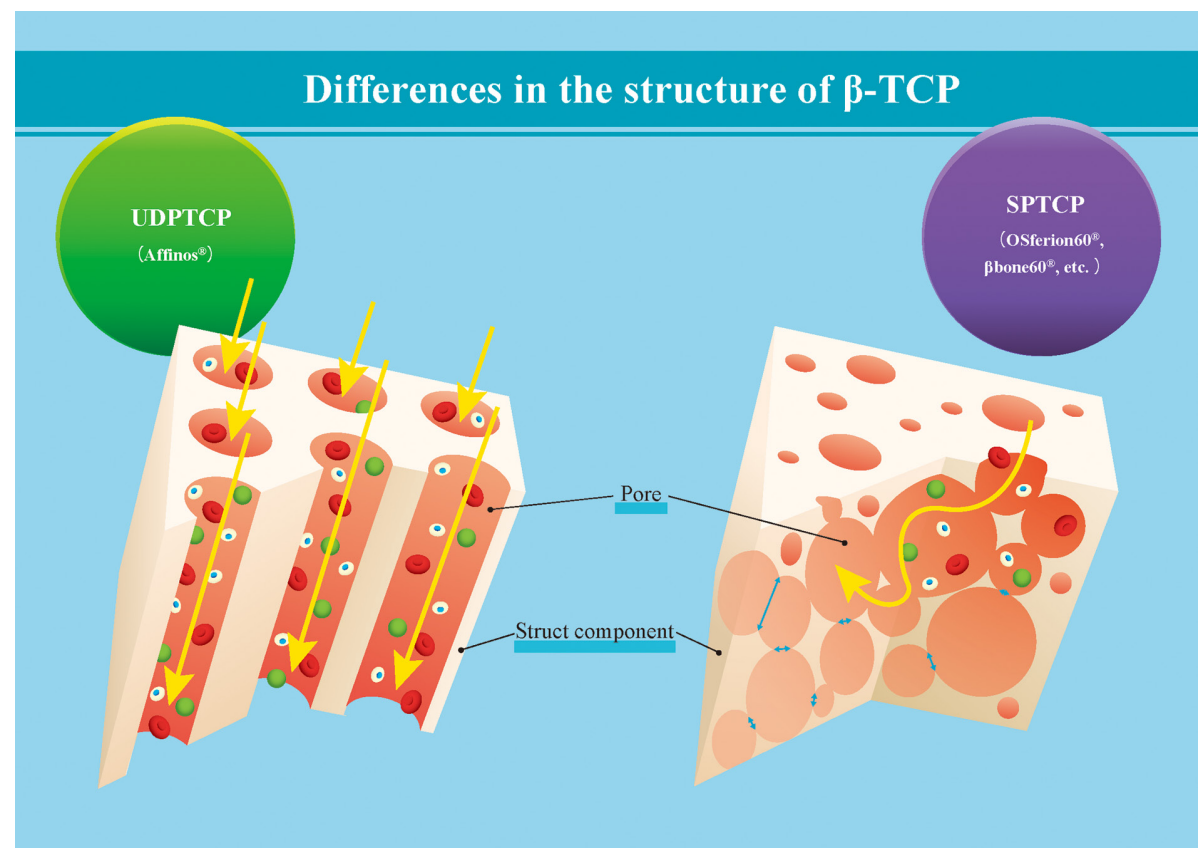

Figure 1 Schematic drawings of soak-loaded PRP onto $\beta$-TCP.

Arrow shows the route through which blood cells enter the PRP. UDPTCP could be penetrated with PRP through the capillary phenomenon, whereas SPTCP could be filled through its spherical pores. $\beta$-TCP: $\beta$-tricalcium phosphate; PRP: platelet-rich plasma; UDPTCP: unidirectional porous $\beta$-TCP; SPTCP: spherical porous $\beta$-TCP. 
ness currently being treated, difficult blood sampling, and/ or anemia.

\section{PRP preparation}

Eight milliliters of anticoagulant (Anticoagulant Citrate Dextrose Solution-A; Zimmer Biomet, Warsaw, Indiana, USA) was aspirated into a $60 \mathrm{ml}$ syringe, and peripheral blood $(52 \mathrm{~mL})$ was collected from the median cubital vein using a 22 $\mathrm{G}$ winged needle. A GPS-III separation tube was filled with blood containing the anticoagulant $(60 \mathrm{~mL})$. The tube was centrifuged at $3,200 \mathrm{rpm}$ for $15 \mathrm{~min}$ at $22-24^{\circ} \mathrm{C}$ using a GPSIII Platelet Concentration System (Zimmer Biomet, Warsaw, Indiana, USA). After removing the platelet-poor plasma (PPP), approximately $6 \mathrm{~mL}$ of PRP was extracted (Figure 2).

\section{Artificial bone}

Affinos $^{\circledR}$ (Kuraray Co., Ltd., Tokyo, Japan) was used as the UDPTCP and $\beta$ bone $60^{\circledR}$ (Zimmer Biomet GK) as the SPTCP. Pieces of UDPTCP and SPTCP that were $8 \mathrm{~mm}$ in diameter were used. Affinos ${ }^{\circledR}$ has 30-250 $\mu \mathrm{m}$ unidirectional pores ${ }^{19)}$ and $\beta$ bone $60^{\circledR}$ has the same structure as OSferion $60^{\circledR}$, which consists of macropores $\geq 100 \mu \mathrm{m}$ and micropores $\leq 5 \mu \mathrm{m}^{25)}$. These parameters meet the criteria to act as scaffold materials for bone regeneration ${ }^{26)}$.

\section{Soaking of artificial bone in PRP}

The $6 \mathrm{~mL}$ of PRP that was extracted previously was dispensed into four individual $5 \mathrm{ml}$ Eppendorf Tubes (Eppendorf) at $1.5 \mathrm{~mL}$ each. The two types of artificial bone were soaked for either 10 seconds or 10 minutes to make a total of four groups (UDTCP 10 seconds; U-10, UDPTCP 10 minutes; U-600, SPTCP 10 seconds; S-10, SPTCP 10 minutes; S-600). After soaking, PRP that adhered to the surface of the artificial bone was wiped with gauze moistened with physiological saline ${ }^{27)}$, and the pieces were placed in vials (Vials With Screw Cap; Nichiden-Rika Glass Co. Ltd., Kobe, Hyogo, Japan) containing 2.5\% glutaraldehyde (Wako, 20\% glutaraldehyde solution diluted with $0.1 \mathrm{~mol} / \mathrm{L}$ phosphate buffer $\mathrm{pH}$ 7.0).

\section{Hematological assessment}

Leukocyte and platelet counts of the peripheral blood and extracted PRP were measured with a cell count analyzer (Sysmex KX-21N; Sysmex Corp., Kobe, Japan) to calculate concentrations as follows. The PAW classification system was used for PRP ${ }^{28)}$.

$[$ leukocyte concentration (times) $]=\frac{[\text { leukocyte count of } \mathrm{PRP}(/ \mu \mathrm{L})]}{[\text { leukocyte count of peripheral blood }(/ \mu \mathrm{L})]}$

\section{Porosity measurement}

Gravimetry was used to calculate porosity ${ }^{26,29)}$. The mass of the artificial bone was measured on an electronic scale (AB204; Mettler Toledo) and the volume was calculated by measuring the distances from the four sides on photographs of artificial bone cross-sections taken to measure the soaked area. The density of the material was $3.07 \mathrm{mg} / \mathrm{mm}^{3}$, which is the theoretical density of $\beta$-TCP.

$[\operatorname{porosity}(\%)]=1-\frac{\left\lfloor\text { artificial bone } \operatorname{density}\left(\mathrm{mg} / \mathrm{mm}^{3}\right)\right\rfloor}{\left[\text { material density }\left(\mathrm{mg} / \mathrm{mm}^{3}\right)\right]}$

The loaded volume capacity was calculated from the porosity obtained with the above formula. The calculations were performed by approximating the specific gravity of the serum and PRP to $1.03\left(\mathrm{mg} / \mathrm{mm}^{3}\right)^{30)}$ and $1.03 \mathrm{mg} / \mathrm{mm}^{3}$,
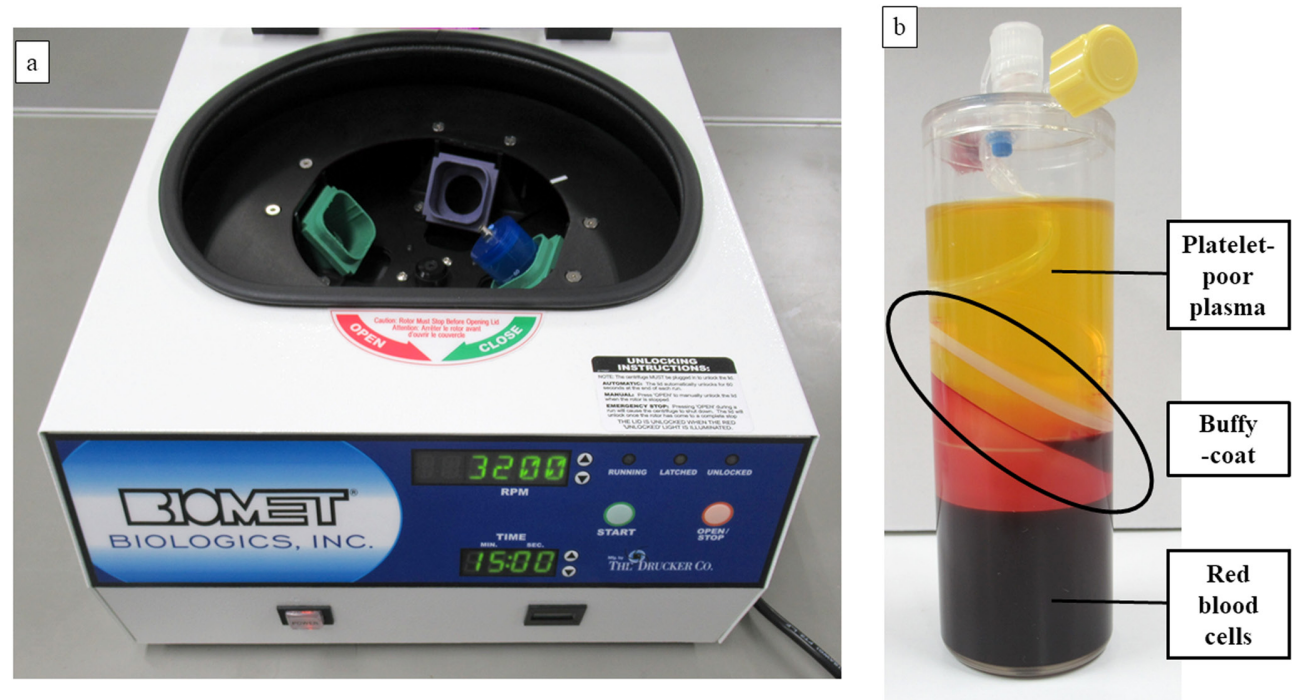

Figure 2 Centrifugation system.

a: Centrifugation at 3,200 rpm for 15 min using the single-spin system (GPS-III Platelet Concentration System; Zimmer Biomet). b: Platelet-rich plasma in GPS-III kit after centrifugation. Buffy-coat float above the red blood cell layer. 
respectively.

[loaded volume capacity $(\mathrm{mg})]=[$ artificial bone volume $\left.\left(\mathrm{mm}^{3}\right)\right] \times[$ porosity $(\%)] \times 1.03\left(\mathrm{mg} / \mathrm{mm}^{3}\right)$

\section{Soak-loaded volume measurement}

The mass was measured on an electronic scale (AB204; Mettler Toledo) and the soak-loaded volume ratio of artificial bone was measured as follows:

[soak loaded volume of artificial bone (mg)] = [artificial bone in a vial with fixative after soaking with PRP (mg)] - [artificial bone before soaking (mg)] - [fixative (mg)] - [vial (mg)]

The soak-loaded volume ratio was calculated as the ratio of the soak-loaded volume to the loaded volume capacity.

$[$ soak loaded volume ratio $(\%)]=\frac{[\text { soak loaded volume }(\mathrm{mg})]}{[\text { loaded volume capacity }(\mathrm{mg})]} \times 100$

\section{Soaked area measurement}

The artificial bone was removed from the fixation tube and sliced using a cutting machine (ISOMET; Buehler, Illinois) parallel to the pores on a plane passing through the center of the cylinder. The cross-section was photographed (PL2; Olympus, Japan, NY1S-MOFA) and printed on high sensitivity paper $210 \times 297 \mathrm{~mm}$ (A4). With the groups blinded, two investigators (TY, HS) traced the outline of the soaked area of the artificial bone in the photographs to create binary images with the soaked area in black and the non-soaked area in white ${ }^{31}$. The area of the cross-section and the soaked area were calculated using ImageJ to semiquantitatively measure the soaked area ratio as follows:
$[$ soaked area ratio $(\%)]=\frac{\left[\text { PRP soaked area }\left(\mathrm{mm}^{2}\right)\right]}{\left[\operatorname{artificial} \text { bone cross section area }\left(\mathrm{mm}^{2}\right)\right]} \times 100$

\section{Statistical analysis}

All results are expressed as the mean \pm standard deviation. Soak-loaded volume ratios were compared using the Tukey-Kramer HSD test, soak-loaded volumes and soaked area ratios with the Games-Howell method, and loaded volume capacities with Welch's t-test. SPSS Statistics software version 25.0 (International Business Machines Co., New York City, NY, USA) was used to calculate statistics and $P<0.05$ was considered a significant difference.

\section{Results}

\section{Hematological assessment}

In the peripheral blood, the mean leukocyte count was $5.3 \pm 1.2 \times 10^{3} / \mu \mathrm{L}$ and the mean platelet count was $21.3 \pm$ $4.7 \times 10^{4} / \mu \mathrm{L}$. In PRP, the mean leukocyte count was $28.1 \pm$ $12.5 \times 10^{3} / \mu \mathrm{L}$ and the mean platelet count was $204.0 \pm 111.1$ $\times 10^{4} / \mu \mathrm{L}$. These values showed a leukocyte and platelet concentration of 5.5 and 9.3 times that of the peripheral blood, respectively (Figure 3). The PAW classification was P4-A $\alpha$.

\section{Porosity measurement}

Porosity calculated by gravimetry was $58.6 \pm 2.1 \%$ for UDPTCP and $61.7 \pm 3.2 \%$ for SPTCP, both of which are similar to the values provided by the companies (UDPTCP $57 \pm 5 \%$, SPTCP 60\%). The PRP loaded volume capacity calculated from these porosity values was $245.3 \pm 19.8 \mathrm{mg}$

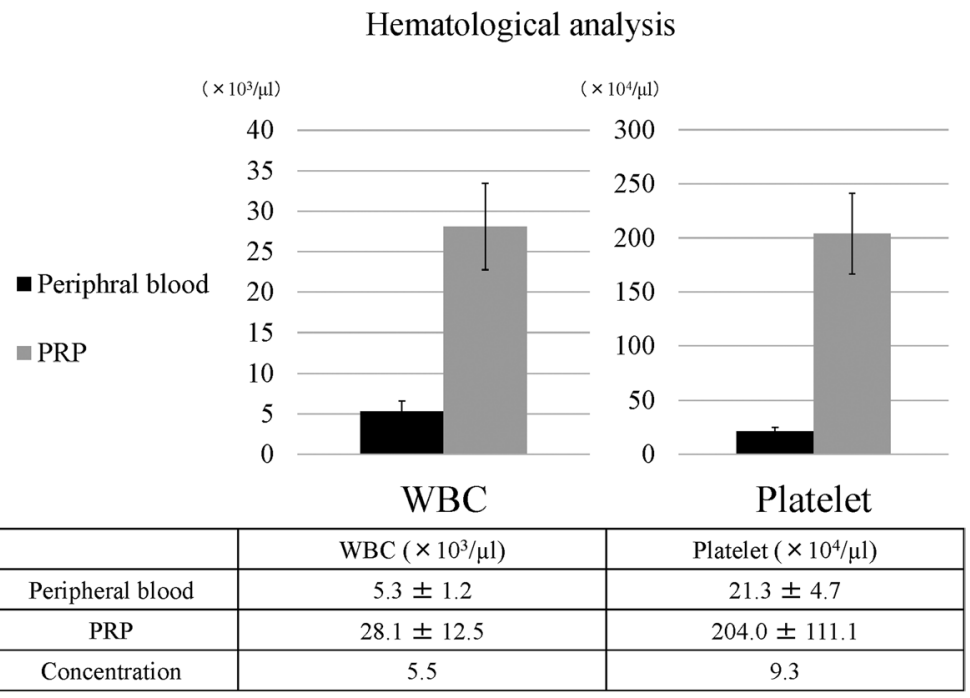

Figure 3 Hematological analysis.

The concentration of leukocytes and platelets is 5.5 and 9.3 times that of the peripheral blood. Data are presented as the mean \pm SD. 


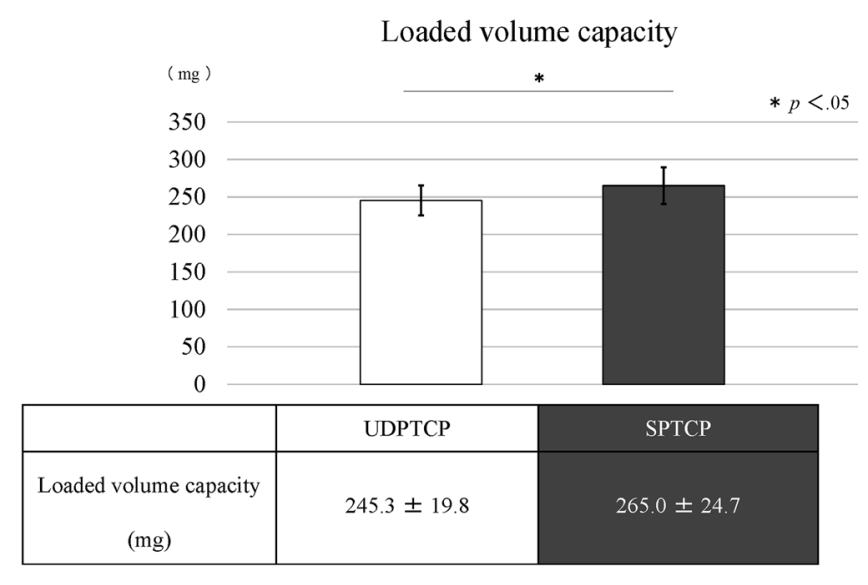

Figure 4 Load volume capacity.

The loaded volume capacity of SPTCP was higher than that of UDPTCP $(P<0.05)$. Data are presented as the mean \pm SD.

for UDPTCP and $265.0 \pm 24.7 \mathrm{mg}$ for SPTCP, with SPTCP being significantly higher (Figure 4).

\section{Soak-loaded volume measurement}

The soak-loaded volumes were $189.5 \pm 7.3 \mathrm{mg}$ for U-10, $182.8 \pm 8.3 \mathrm{mg}$ for $\mathrm{U}-600,91.7 \pm 28.9 \mathrm{mg}$ for $\mathrm{S}-10$, and 120.0 $\pm 15.3 \mathrm{mg}$ for S-600. When comparing the soaking times (U-10 vs. S-10, U-600 vs. S-600), UDPTCP was significantly higher than SPTCP. When comparing the artificial bone types, there was no significant difference between U-10 and U-600, although S-600 was significantly higher than S-10.

The soak-loaded volume ratios were $75.6 \pm 6.4 \%$ for $\mathrm{U}-10,76.8 \pm 4.9 \%$ for $\mathrm{U}-600,34.0 \pm 9.1 \%$ for $\mathrm{S}-10$, and 46.0 $\pm 7.0 \%$ for S- 600 . When comparing U-10 to S-10 and U-600 to S-600, UDPTCP was significantly higher for both times. When comparing the artificial bone types, there was no significant difference between U-10 and U-600, although S-600 was significantly higher than S-10 (Figure 5).

\section{Soaked area measurement}

The soaked area ratios were $100 \%$ for U-10 and U-600, $65.0 \pm 5.4 \%$ for S-10, and $67.9 \pm 9.1 \%$. for S- 600 . When comparing the soaking times (U-10 vs. S-10, U-600 vs. S-600), UDPTCP was significantly higher. When comparing the artificial bone types, there were no significant differences between U-10 and U-600, or between S-10 and S-600 (Figure 6).

\section{Discussion}

In this study, UDPTCP was able to soak-load PRP faster, and in larger amounts, than SPTCP. Previous studies have reported that loading $\beta$-TCP with PRP promotes osteogenesis $^{7,8)}$. A histological study on the femoral bone defect mouse model confirmed that polycaprolactone- $20 \%$ tricalcium phosphate (PCL-TCP) soak-loaded with $60 \mu \mathrm{L}$ of leukocyte-poor PRP (LP-PRP) promoted osteogenesis after 12 weeks $^{7}$. Radiographic and histological evaluations in a study on a radial defect rabbit model showed that SPTCP soak-loaded with bone marrow mesenchymal stem cells (BM-MSCs) and $200 \mu \mathrm{L}$ of leukocyte-rich PRP (LR-PRP) promoted osteogenesis after both 4 and 8 weeks ${ }^{8}$. In addition, administering granulated artificial bone mixed with activated PRP promotes osteogenesis ${ }^{9}{ }^{10}$. In a study on a tibial fracture rabbit model, administering LR-PRP mixed with SPTCP promoted osteogenesis as seen through radiological, histological, and quantitative computed tomographic images after 12 weeks ${ }^{9}$. However, in an RCT of maxillary sinus defects, administration of $\beta$-TCP mixed with LP-PRP did not promote osteogenesis ${ }^{10}$. None of these studies quantified the amount of PRP that was loaded onto $\beta$-TCP.

The PRP used in the present study was leukocyte-rich PRP, which was classified as P4-A $\alpha$ according to PAW classification, and was obtained using a GPS-III system. In previous reports, PRP prepared from GPS-III systems had leukocyte concentrations of 3-5 times and platelet concentrations of 3-8 times those of the peripheral blood ${ }^{32-35)}$, which are similar to our results (Table 1). Marx et al. ${ }^{14)}$ reported on PRP therapy for mandibular bone defects, stating that platelet counts of $100 \times 10^{4} / \mu \mathrm{L}$ and concentrations of 4-5 times that of the peripheral blood were the minimum necessary conditions to promote osteogenesis. The PRP used in the present study met these criteria.

The loaded volume capacity of artificial bone in a cylinder ( $8 \mathrm{~mm}$ in diameter by $8 \mathrm{~mm}$ in length) was significantly higher with SPTCP than with UDPTCP. Therefore, to compare PRP soak-loaded volumes based on the presence or absence of a unidirectional porous structure and different soaking times, we did not calculate soak-loaded volume but rather the soak-loaded volume ratio, and not the soaked area but the soaked area ratio.

The higher soak-loaded volume ratios regardless of soaking time suggest that the capillarity phenomenon created by the unidirectional porous structure ${ }^{19)}$ enabled a large amount of PRP to be loaded in a short time. The fact that soak-loaded volume ratios reached a maximum at $75-$ $77 \%$ may indicate that, because PRP was in a liquid state, some of it leaked from the pores when the artificial bone was removed from the PRP. Regarding the soaked area in the cross-section, PRP soaked the UDPTCP cross-section uniformly, indicating that the pores were filled with PRP during soaking. In contrast, the soak-loaded volume ratio of SPTCP was lower than $50 \%$, even after soaking for 10 minutes, and penetration at the center of the cross-sections ceased at approximately $65-68 \%$. These results show that PRP did not reach deeply into SPTCP, which may be due to differences in the structures of $\beta-\mathrm{TCP}^{6}$ ) and interference of air remaining in the pores ${ }^{36}$. 


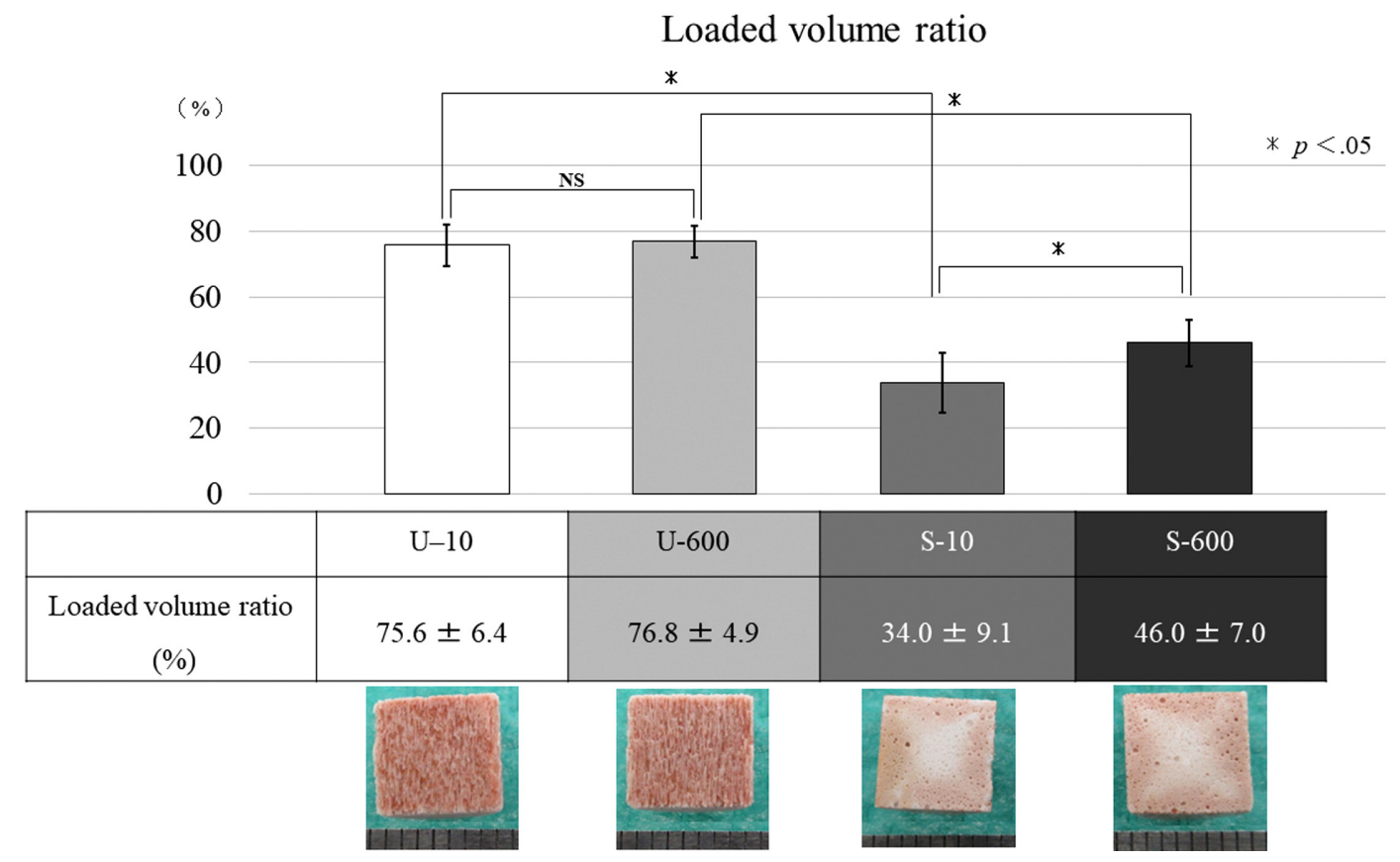

Figure 5 Soak-loaded volume ratio.

The UDPTCP soak-loaded volume ratio was significantly higher than $\operatorname{SPTCP}(P<0.05)$. Data are presented as the mean $\pm \mathrm{SD}$.

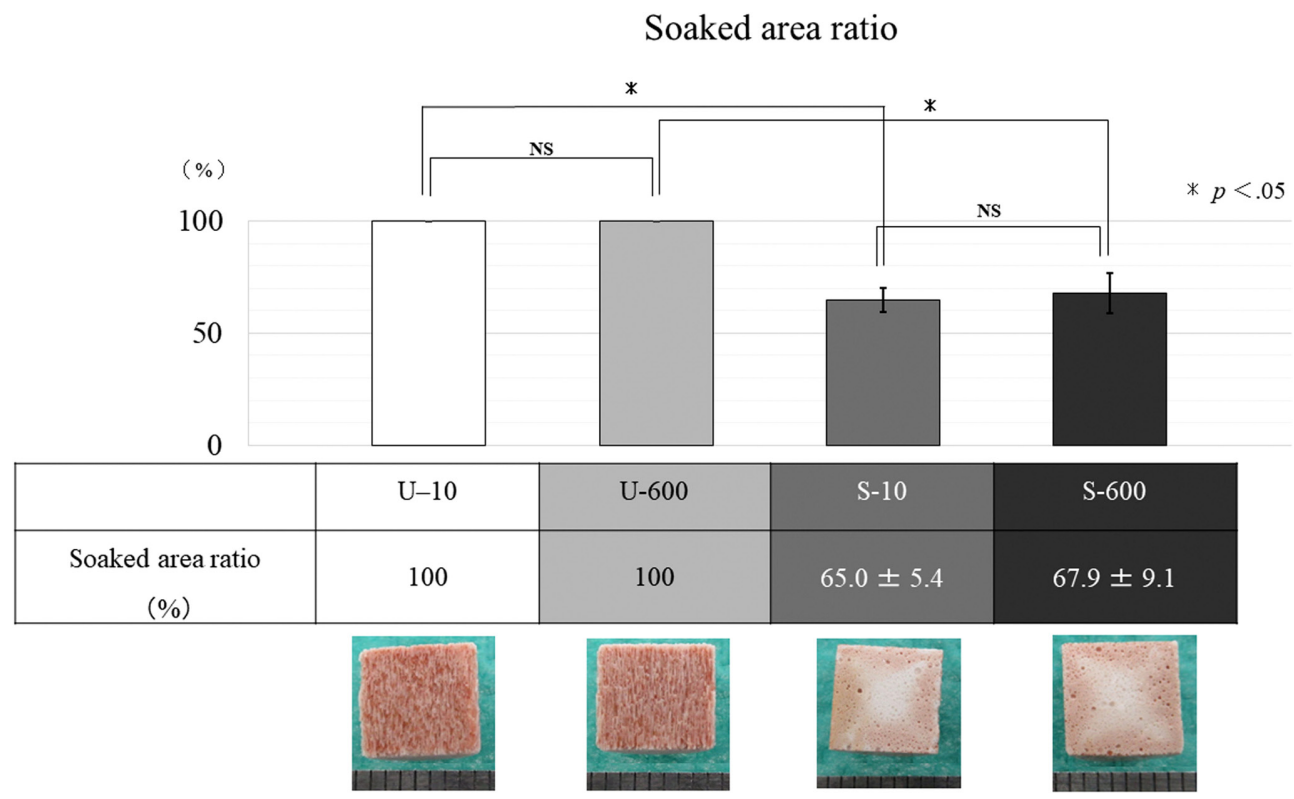

Figure 6 Soaked area ratio.

The UDPTCP soaked area ratio was significantly higher than SPTCP $(P<0.05)$. Data are presented as the mean \pm SD.

Table 1 PRP concentration rates with the GPS-III system

\begin{tabular}{lccccc}
\hline & Fitzpatrick & Castillo & Sundman & Eppley & Present study \\
Leukocyte concentration & $3-5$ & 5.4 & 4.2 & 5.4 & 5.5 \\
Platelet concentration & $3-6$ & 2.1 & 3.8 & 8.1 & 9.3 \\
\hline
\end{tabular}

PRP: Platelet-rich plasma. 
The significantly higher soak-loaded volume ratio of S-600 compared with S-10 suggests that more PRP can be soak-loaded onto SPTCP if it is soaked for a longer time. However, PRP reportedly releases $70 \%$ of its growth factors 10 min after activation and $100 \% 1$ hour after activation ${ }^{28)}$. Once the $\beta$-TCP is soaked with PRP, PRP may be activated and transformed into a gel after $10 \mathrm{~min}$. Thereafter, platelets cannot reach deep into the $\beta$-TCP. Therefore, when using artificial bone soak-loaded with PRP in clinical practice, it would be desirable to quickly load and administer the artificial bone with PRP. The soak-loaded volume of U-10 was significantly higher than that of S-600, which indicates that, since UDPTCP can be soak-loaded with PRP faster and in larger amounts than SPTCP, it could serve as a novel osteogenic composite.

This study has some limitations. First, this study did not evaluate osteogenesis in vivo. It is important to evaluate whether the rate of PRP loading onto the $\beta$-TCP affects its osteo-inductive activity. Further studies are needed to evaluate bone union by comparing UDPTCP and SPTCP soak-loaded with PRP in vivo, such as in a lumbar interbody fusion cage. Second, the number of platelets and the amount of growth factors that were loaded are unknown. Platelets ( $2 \mu \mathrm{m}$ in diameter) are smaller than red blood cells (RBCs; $7 \mu \mathrm{m}$ in diameter $)^{30}$. The soaked area in the cross-section appeared red, suggesting the presence of RBCs; therefore, loaded platelets may be present in the soaked area. In addition to the quantitative studies of PRP, further studies are needed to investigate which growth factors, and at what concentration, are effective for osteogenesis.

\section{Conclusion}

UDPTCP can soak-load PRP faster and in larger amounts than SPTCP. Further studies are necessary to determine the optimal PRP concentration that promotes bone formation. The basic data from our study could be used as a baseline for future clinical applications and studies in orthopedic surgery such as lumbar interbody fusion and nonunion surgeries.

Conflict of interests: One author is affiliated with the Department of Regenerative Medicine for Musculoskeletal System, Faculty of Medicine, University of Tsukuba, Tsukuba, Japan. This department is an endowment department, supported by an unrestricted grant from Zimmer Biomet GK. No commercial party has a direct financial interest in the results of the research. Supporting this article has or will confer a benefit to the other authors or on any organization with which the other authors are associated.

\section{Acknowledgement}

The authors would like to thank Kuraray Co., Ltd. for supplying $\beta$-TCP and Zimmer Biomet GK for supplying $\beta$-TCP and Platelet concentrate separation kits.

\section{References}

1. Kim DH, Rhim R, Li L, et al. Prospective study of iliac crest bone graft harvest site pain and morbidity. Spine J 2009; 9: 886-892. [Medline] [CrossRef]

2. Myeroff C, Archdeacon M. Autogenous bone graft: donor sites and techniques. J Bone Joint Surg Am 2011; 93: 2227-2236. [Medline] [CrossRef]

3. Urabe K, Itoman M, Toyama Y, et al. Current trends in bone grafting and the issue of banked bone allografts based on the fourth nationwide survey of bone grafting status from 2000 to 2004. J Orthop Sci 2007; 12: 520-525. [Medline] [CrossRef]

4. Barradas AMC, Yuan H, van Blitterswijk CA, et al. Osteoinductive biomaterials: current knowledge of properties, experimental models and biological mechanisms. Eur Cell Mater 2011; 21: 407-429, discussion 429. [Medline] [CrossRef]

5. Goshima J, Goldberg VM, Caplan AI. The osteogenic potential of culture-expanded rat marrow mesenchymal cells assayed in vivo in calcium phosphate ceramic blocks. Clin Orthop Relat Res 1991; 298-311. [Medline]

6. Tanaka M, Haniu H, Kamanaka T, et al. Physico-chemical, in vitro, and in vivo evaluation of a 3D unidirectional porous hydroxyapatite scaffold for bone regeneration. Materials (Basel) 2017; 10: 33-45. [Medline] [CrossRef]

7. Rai B, Ho KH, Lei Y, et al. Polycaprolactone- $20 \%$ tricalcium phosphate scaffolds in combination with platelet-rich plasma for the treatment of critical-sized defects of the mandible: a pilot study. J Oral Maxillofac Surg 2007; 65: 2195-2205. [Medline] [CrossRef]

8. Yu T, Pan H, Hu Y, et al. Autologous platelet-rich plasma induces bone formation of tissue-engineered bone with bone marrow mesenchymal stem cells on beta-tricalcium phosphate ceramics. J Orthop Surg Res 2017; 12: 178. [Medline] [CrossRef]

9. Szponder T, Wessely-Szponder J, Sobczyńska-Rak A, et al. Application of platelet-rich plasma and tricalcium phosphate in the treatment of comminuted fractures in animals. In Vivo 2018; 32: 1449-1455. [Medline] [CrossRef]

10. Cömert Kılıç S, Güngörmüş M, Parlak SN. Histologic and histomorphometric assessment of sinus-floor augmentation with beta-tricalcium phosphate alone or in combination with pure-platelet-rich plasma or platelet-rich fibrin: a randomized clinical trial. Clin Implant Dent Relat Res 2017; 19: 959-967. [Medline] [CrossRef]

11. Giannoudis PV, Einhorn TA, Marsh D. Fracture healing: the diamond concept. Injury 2007; 38(Suppl 4): S3-S6. [Medline] [CrossRef]

12. Gomez-Salazar M, Gonzalez-Galofre ZN, Casamitjana J, et al. Five decades later, are mesenchymal stem cells still relevant? Front Bioeng Biotechnol 2020; 8: 148. [Medline] [CrossRef]

13. Wong DA, Kumar A, Jatana S, et al. Neurologic impairment from ectopic bone in the lumbar canal: a potential complication of off-label PLIF/TLIF use of bone morphogenetic protein-2 (BMP-2). Spine J 2008; 8: 1011-1018. [Medline] [CrossRef]

14. Marx RE. Platelet-rich plasma (PRP): what is PRP and what is not PRP? Implant Dent 2001; 10: 225-228. [Medline] [CrossRef] 
15. Anitua E, Tejero R, Zalduendo MM, et al. Plasma rich in growth factors promotes bone tissue regeneration by stimulating proliferation, migration, and autocrine secretion in primary human osteoblasts. J Periodontol 2013; 84: 1180-1190. [Medline] [CrossRef]

16. Marx RE, Carlson ER, Eichstaedt RM, et al. Platelet-rich plasma: growth factor enhancement for bone grafts. Oral Surg Oral Med Oral Pathol Oral Radiol Endod 1998; 85: 638-646. [Medline] [CrossRef]

17. Sakane M, Noguchi H, Ochiai N, et al. Novel scaffold for bone tissue engineering: unidirectional porous hydroxyapatite. In: Nusem I, editor. Bone Grafts: Procedures, Complications and Alternatives. Nova Science Publishers, Hauppauge, 2013; 29-40.

18. Iwasashi M, Funayama $\mathrm{T}$, Watanabe $\mathrm{A}$, et al. Bone regeneration and remodeling within a unidirectional porous hydroxyapatite bone substitute at a cortical bone defect site: histological analysis at one and two years after implantation. Materials (Basel) 2015; 8: 4884-4894. [Medline] [CrossRef]

19. Makihara T, Sakane M, Yamazaki M, et al. The balance between bone formation and material resorption in unidirectional porous $\beta$-tricalcium phosphate implanted in a rabbit tibia. Key Eng Mater 2016; 696: 177-182. [CrossRef]

20. Noguchi H, Funayama T, Koda M, et al. A unidirectional porous beta-tricalcium phosphate material (Affinos ${ }^{\mathbb{E}}$ ) for reconstruction of bony defects after excision of fibular bone for spinal surgery graft. J Clin Neurosci 2019; 66: 71-76. [Medline] [CrossRef]

21. Izawa S, Funayama T, Yamazaki M, et al. The use of unidirectional porous $\beta$-tricarcium phosphate in surgery for calcaneal fractures: a report of four cases J Foot Ankle Res 2017; 10: 2.

22. Kumagai H, Iwasashi M, Funayama T, et al. Surgical repair of acetabular fracture using unidirectional porous $\beta$-tricalcium phosphate. Case Rep Orthop 2019; 2019: 6860591 10.1155/2019/6860591. [Medline]

23. Chazono M, Tanaka T, Komaki H, et al. Bone formation and bioresorption after implantation of injectable $\beta$-tricalcium phosphate granules-hyaluronate complex in rabbit bone defects. J Biomed Mater Res A 2004; 70: 542-549. [Medline] [CrossRef]

24. Ogose A, Kondo N, Umezu H, et al. Histological assessment in grafts of highly purified beta-tricalcium phosphate $\left(\mathrm{OSferion}^{\circledR}\right)$ in human bones. Biomaterials 2006; 27: 1542-1549. [Medline] [CrossRef]

25. Tanaka T, Kumagae Y, Saito M, et al. Bone formation and resorption in patients after implantation of $\beta$-tricalcium phosphate blocks with $60 \%$ and $75 \%$ porosity in opening-wedge high tibial osteotomy. J Biomed Mater Res B Appl Biomater 2008; 86: 453-459. [Medline] [CrossRef]

26. Karageorgiou V, Kaplan D. Porosity of 3D biomaterial scaffolds and osteogenesis. Biomaterials 2005; 26: 5474-5491. [Medline] [CrossRef]

27. Japanese Industrial Standard, R 1634: 1998. Test methods for density and apparent porosity of fine ceramics, 6.4 Mass of water-saturated test specimen.

28. DeLong JM, Russell RP, Mazzocca AD. Platelet-rich plasma: the PAW classification system. Arthroscopy 2012; 28: 998-1009. [Medline] [CrossRef]

29. Hu Y, Grainger DW, Winn SR, et al. Fabrication of poly (alpha-hydroxy acid) foam scaffolds using multiple solvent systems. J Biomed Mater Res 2002; 59: 563-572. [Medline] [CrossRef]

30. Alsousou J, Thompson M, Hulley P, et al. The biology of platelet-rich plasma and its application in trauma and orthopaedic surgery: a review of the literature. J Bone Joint Surg Br 2009; 91: 987-996. [Medline] [CrossRef]

31. Mikami S, Chiba S, Hojo H, et al. Immunocytochemical studies on the pituitary pars distalis of the Japanese long-fingered bat, Miniopterus schreibersii fuliginosus. Cell Tissue Res 1988; 251: 291-299. [Medline] [CrossRef]

32. Fitzpatrick J, Bulsara MK, McCrory PR, et al. Analysis of platelet-rich plasma extraction variations in platelet and blood components between 4 common commercial kits. Orthop J Sports Med 2017; 5: 2325967116675272. [Medline] [CrossRef]

33. Castillo TN, Pouliot MA, Kim HJ, et al. Comparison of growth factor and platelet concentration from commercial platelet-rich plasma separation systems Am J Sports Med 2011; 39: 266-271. [Medline] [CrossRef]

34. Sundman EA, Cole BJ, Fortier LA. Growth factor and catabolic cytokine concentrations are influenced by the cellular composition of platelet-rich plasma. Am J Sports Med 2011; 39: 2135-2140. [Medline] [CrossRef]

35. Eppley BL, Woodell JE, Higgins J. Platelet quantification and growth factor analysis from platelet-rich plasma: implications for wound healing. Plast Reconstr Surg 2004; 114: 1502-1508. [Medline] [CrossRef]

36. Hasegawa T, Miwa M, Sakai Y, et al. Efficient cell-seeding into scaffolds improves bone formation. J Dent Res 2010; 89: 854-859. [Medline] [CrossRef] 